\section{Posições oficiais 2008 da Sociedade Brasileira de Densitometria Clínica (SBDens)}

\author{
2008 Official positions of the Brazilian Society \\ for Clinical Densitometry - SBDens
}

Cynthia M. A. Brandão $0^{1,2,3}$, Bruno Muzzi Camargos ${ }^{1,4}$, Cristiano Augusto Zerbini ${ }^{5,6}$, Pérola Grinberg Plapler $2,7,8$, Laura Maria de Carvalho Mendonça ${ }^{2,5,6}$, Ben-Hur Albergaria ${ }^{2,10}$, Marcelo Medeiros Pinheiro ${ }^{1}$, Mirley do Prado ${ }^{1,12}$, Sergio Ragi Eis ${ }^{1,13}$

\section{RESUMO}

A evolução dos métodos de avaliação da massa óssea trouxe diferentes tecnologias, modos de aquisição de imagens, bancos de dados de referência, terminologias, critérios diagnósticos fez com que a International Society for Bone Densitometry (ISCD) tomasse a iniciativa de promover reuniões periódicas de consenso, a última em 2007. A Sociedade Brasileira de Densitometria Clínica (SBDens), com apoio de várias sociedades brasileiras ligadas ao estudo da saúde óssea, reuniu diversos especialistas para discutir as propostas da ISCD e validar a aplicação destas normas à população brasileira. A reunião de Posições Oficiais da SBDens produziu um documento extremamente útil para a compreensão e interpretação da densitometria e de outros métodos de avaliação da massa óssea. Arq Bras Endocrinol Metab. 2009;53(1):107-112.

Descritores

Densitometria; densidade mineral óssea; osteoporose; risco de fratura; absorciometria duplo-energética, posições oficiais

\section{ABSTRACT}

With the evolution of bone densitometry, differences in technologies, acquisition techniques, reference databases, reporting methods, diagnostic criteria and terminology have developed and the International Society for Clinical Densitometry (ISCD) periodically holds Position Development Conferences, the latest in 2007. The Brazilian Society for Clinical Densitometry (SBDens), with support from many Brazilian societies interested in bone health, gathered numerous specialists to discuss the ISCD proposals and to evaluate the validity of the extension of those norms to Brazilian population. The SBDens reunion of consensus made a very utile document to help the understanding and interpretation of bone densitometry and other methods of bone assessment. Arq Bras Endocrinol Metab. 2009;53(1):107-112.

Keywords

Densitometry; bone mineral density; osteoporosis; risk of fracture; dual-energy X-ray absorptiometry; official positions

\author{
' Sociedade Brasileira de \\ Densitometria Clínica (SBDens) \\ 2 Sociedade Brasileira para \\ o Estudo do Metabolismo Ósseo \\ e Mineral (SOBEMOM) \\ ${ }^{3}$ Disciplina de Endocrinologia da \\ Universidade Federal de São Paulo \\ (Unifesp), São Paulo, SP, Brasil \\ ${ }^{4}$ Hospital Mater Dei, Universidade \\ Federal de Minas Gerais, \\ Belo Horizonte, MG, Brasil \\ ${ }^{5}$ Sociedade Brasileira de \\ Reumatologia (SBR) \\ ${ }^{6}$ Sociedade Brasileira de \\ Osteoporose (SOBRAO) \\ ${ }^{7}$ Instituto de Ortopedia e \\ Traumatologia, Hospital das Clínicas, \\ Faculdade de Medicina, \\ Universidade de São Paulo \\ (IOT-HC-FMUSP), São Paulo, SP, Brasil \\ ${ }^{8}$ Associação Brasileira de Medicina \\ Física e Reabilitação (SPFR) \\ ${ }^{9}$ Sociedade Brasileira de \\ Osteoporose (SOBRAO) \\ ${ }^{10}$ Disciplina de Ginecologia, \\ Universidade Federal do \\ Espírito Santo, Vitória, ES, Brasil \\ "Disciplina de Reumatologia, \\ Unifesp, São Paulo, SP, Brasil \\ ${ }^{12}$ Colégio Brasileiro de \\ Radiologia (CBR) \\ ${ }^{13}$ Sociedade Brasileira de \\ Ortopedia e Traumatologia (SBOT)
}

Correspondência para

Cynthia M. Álvares Brandão Rua Pelotas, 562

04012002 São Paulo SP

cynthia.brandao@fleury.com.br

Recebido em 25/Dez/2008

Aceito em 12/Jan/2009

\section{INTRODUÇÃO}

$\mathrm{E}$ $\mathrm{m}$ todo o mundo, diversas entidades médicas têm Ucomo rotina a elaboração periódica de documentos que orientam a prática clínica, fundamentados em criteriosos levantamentos da literatura médica e, sobretudo, nas mais recentes evidências científicas. Com este mesmo intuito, a Sociedade Brasileira de Densitometria Clínica (SBDens) promoveu, em outubro de 2008, sua Segunda Reunião de Desenvolvimento das Posições Oficiais. O evento reuniu médicos de diferentes especialidades que discutiram tópicos referentes a métodos não-invasivos de avaliação da massa óssea propostos pela International Society for Clinical Densitometry (ISCD). Sob coordenação científica, cinco relatores discorreram sobre os temas propostos e um texto final foi elaborado, sendo, então, submetido à aprovação dos participantes, todos membros da SBDens, por meio do voto individual.

O resultado deste trabalho está detalhado em um documento que pode ser acessado em sua íntegra no site da entidade (www.sbdens.org.br), e que passa a representar, até 
futura revisão, as Posições Oficiais da Sociedade Brasileira de Densitometria 2008 (SBDens), apoiadas por entidades afins, quais sejam, Sociedade Brasileira para o Estudo do Metabolismo Ósseo e Mineral (Sobemom), Sociedade Brasileira de Osteoporose (Sobrao), Colégio Brasileiro de Radiologia (CBR), Sociedade Brasileira de Reumatologia (SBR), Comitê de Doenças Osteometabólicas da Sociedade Brasileira de Ortopedia (SBOT), Federação Brasileira de Sociedades de Ginecologia e Obstetrícia (Febrasgo), Sociedade Brasileira de Endocrinologia e Metabolismo (SBEM), Sociedade Brasileira de Biologia e Medicina Nuclear (SBBMN) e Sociedade Brasileira de Geriatria e Gerontologia (SBGG). O objetivo desta publicação é divulgar mais amplamente os princípios da realização e da interpretação dos métodos de avaliação da massa óssea, em um texto sintético e esquemático, ressaltando particularmente os tópicos de maior interesse clínico.

\section{INDICAÇÕES PARA AVALIAÇÃO DA DENSIDADE MINERAL ÓSSEA}

- Mulheres com idade $\geq$ a 65 anos e homens com idade $\geq$ a 70 anos.

- Mulheres acima de 40 anos, na transição menopausal (1-2) e homens acima de 50 anos de idade, com fatores de risco*.

- Adultos com antecedente de fratura por fragilidade, condição clínica ou uso de medicamentos associados à baixa massa óssea ou perda óssea.

- Indivíduos para os quais são consideradas intervenções farmacológicas para osteoporose.

- Indivíduos em tratamento para osteoporose, para monitoramento de sua eficácia.

- Indivíduos que não estejam sob tratamento, porém nos quais a identificação de perda de massa óssea possa determinar a indicação do tratamento.

- Mulheres interrompendo terapia hormonal (TH).

\section{BASE DE DADOS DE REFERÊNCIA}

A base de dados NHANES III (National Health and Nutrition Examination Survey III) (12) deve ser utilizada para derivação do T-escore (número de desviospadrão da média de adultos jovens), particularmente no fềmur proximal.

*Os fatores mais comumente associados ao maior risco de fraturas, em inúmeras populações estudadas (3-10), inclusive no Brasil (11), são: sexo feminino, raça branca idade acima de 60 anos, peso corporal abaixo de $55 \mathrm{~kg}$, fratura prévia por baixo impacto (decorrente de trauma semelhante ou inferior à queda da própria altura) após os 40 anos de idade, história familiar de fratura após os 50 anos de idade em parentes de primeiro grau, tabagismo atual, corticoterapia prolongada (dose diária de prednisona acima de $5 \mathrm{mg}$, ou equivalente, por tempo superior a três meses), uso regular de bebidas alcoólicas (acima de duas doses diárias) e sedentarismo.
- Tanto para mulheres quanto para homens deve ser utilizada uma base de dados de normalidade de caucasianos, para todos os grupos étnicos (não ajustar para a etnia).

\section{DIAGNÓSTICO DENSITOMÉTRICO CENTRAL}

- Os critérios da OMS (12), de 1994, determinam que o diagnóstico de osteoporose pode ser feito em mulheres menopausadas e homens com idade $>50$ anos, se houver um T-escore igual ou inferior a $(-2,5)$ em qualquer um dos seguintes sítios ósseos, mesmo na ausência de histórico de fratura osteoporótica: fềmur proximal (colo femoral e fềmur total), coluna lombar (Ll-L4) e rádio 33\% (diáfise do rádio, com predomínio de osso cortical).

- A osteopenia é definida com valores de T-escore entre $(-1,01)$ e $(-2,49)$.

- O rádio 33\% é o único sítio ósseo periférico que pode ser utilizado para fins diagnósticos.

- O rádio 33\% deve ser realizado quando a coluna lombar ou o fêmur proximal não puderem ser medidos ou interpretados nos pacientes com diagnóstico de hiperparatireoidismo, e nos obesos com peso acima do limite do equipamento DXA utilizado.

- Os critérios diagnósticos da OMS podem ser aplicados às mulheres na transição menopausal.

\section{REGIÕES DE INTERESSE NA COLUNA LOMBAR PA}

- Deve ser utilizado o segmento Ll-L4. Quanto maior a área analisada, maior a reprodutibilidade.

- Devem ser utilizadas todas as vértebras avaliáveis (Ll, L2, L3 e L4) e excluídas apenas uma ou duas vértebras que estejam afetadas por alterações morfológicas e estruturais ou de artefatos. Não é possível fazer o diagnóstico considerando-se apenas uma vértebra.

- Se apenas uma vértebra lombar for avaliável, depois de excluídas as demais, o diagnóstico deverá basear-se em outro sítio esquelético válido.

- Condições que justificam a exclusão de vértebras: alterações anatômicas, manipulação cirúrgica e processos osteodegenerativos que resultem diferença de mais de um desvio-padrão (T-escore) entre a vértebra em questão e a adjacente.

- O exame lateral da coluna lombar não deve ser usado para fins diagnósticos, embora possa ser útil no monitoramento.

\section{REGIÕES DE INTERESSE NO FÊMUR PROXIMAL}

- O colo femoral e a região do fềmur total devem ser avaliadas. 
- Ambos os fềmures podem ser medidos.

- As regiões de Ward e o trocânter não devem ser usados para diagnóstico.

- Não existem dados que justifiquem a utilização da média dos T-escores de ambos os fêmures para o diagnóstico.

- Para monitoramento, a região de interesse fêmur total deve ser preferida.

\section{REGIÕES DE INTERESSE NO ANTEBRAÇO}

- O rádio 33\% (rádio $1 / 3$ ) do antebraço não dominante, região de predomínio de osso cortical, deve ser utilizado para diagnóstico.

\section{AVALIAÇÃO DO RISCO DE FRATURAS}

- Os valores de corte de T-escore dos critérios diagnósticos da OMS só podem ser aplicados à densitometria óssea.

- Para avaliação do risco de fraturas, outras técnicas além da densitometria por DXA podem ser utilizadas.

\section{DENSITOMETRIA EM MULHERES NA MENACME E HOMENS COM MENOS DE 50 ANOS}

- Deve ser usado o Z-escore (número de desvios-padrão da média de uma população do mesmo sexo e raça do indivíduo).

- Z-escore igual ou inferior a -2.0 desvios-padrão é definido como "abaixo da faixa esperada para a idade" e um Z-escore acima de -2.0 desvios-padrão deve ser classificado como "dentro dos limites esperados para a idade".

- O termo osteopenia pode ser utilizado, mas "baixa massa óssea para a idade" deve ser preferido, pois indivíduos jovens com baixa DMO não apresentam, necessariamente, elevado risco de fraturas.

- Z-escores devem ser definidos para população específica sempre que existirem referências adequadas. Para propósito do cálculo do Z-escore, a etnia definida pelo próprio paciente deve ser utilizada.

- A osteoporose não pode ser diagnosticada em homens saudáveis com menos de 50 anos ou mulheres saudáveis com menos de 40 anos de idade fundamentando-se isoladamente em critérios de DMO; no entanto, se houver uma causa secundária estabelecida (por exemplo, uso crônico de corticosteróides) o termo osteoporose e osteopenia podem ser utilizados.

\section{MEDIDAS SERIADAS DE DMO}

- Medidas seriadas de DMO podem demonstrar a eficácia do tratamento, detectando estabilidade ou ganho de DMO.

- Medidas seriadas de DMO podem identificar ausência de resposta ao tratamento, caso seja observada perda de DMO, sugerindo necessidade de ser reavaliada a opção terapêutica, bem como a necessidade de se investigar a presença de causas secundárias de osteoporose e perda óssea.

- A MVS deve ser utilizada para avaliar as variações observadas entre medidas seriadas da DMO (13).

- O período de intervalo entre exames deve ser determinado de acordo com a condição clínica de cada paciente. Habitualmente, um ano após o início ou mudança do tratamento, nova medida de DMO é apropriada. Maiores intervalos deverão ser observados quando a eficácia terapêutica já estiver estabelecida.

- Em condições associadas à perda óssea rápida, como tratamento com glicocorticóides, exames com periodicidade menor conseguem identificar alterações significativas.

\section{AVALIAÇÕES DE PRECISÃO}

- Cada serviço de densitometria deve determinar seu próprio erro de precisão ou coeficiente de variação $(\mathrm{CV} \%)$, com base em estudo in vivo, com pacientes que representem a população geral, e calcular o valor da MVS. O $\mathrm{CV} \%$ indicado pelo fabricante não deve ser considerado com finalidade clínica.

- Novo estudo de precisão deve ser realizado sempre que novo equipamento DXA for instalado, quando o nível de habilidade do operador for modificado ou a cada dois anos.

\section{INDICAÇÕES DE DENSITOMETRIA EM OSTEOPOROSE INDUZIDA POR GLICOCORTICÓIDES}

- Homens e mulheres em uso ou com previsão de uso de glicocorticóides orais por mais de três meses, pulsoterapia endovenosa ou glicocorticóides inalatórios em altas doses.

\section{INDICAÇõES DE MONITORAMENTO EM PACIENTES USUÁRIOS CRÔNICOS DE GLICOCORTICÓIDES}

- A cada seis meses no primeiro ano.

- A cada 12 meses após a estabilização da DMO. 


\section{EXAME DE AVALIAÇÃO DE FRATURAS VERTEBRAIS (VFA)}

Quando a medida da DMO for indicada, a realização complementar de estudo de VFA deve ser considerada nas seguintes condições clínicas, particularmente nos idosos e nos pacientes com diagnóstico prévio de osteoporose.

- Pacientes com perda documentada $(-2 \mathrm{~cm})$ ou relatada $(-4 \mathrm{~cm})$ de estatura desde a idade adulta jovem.

- Pacientes com antecedente de fraturas por fragilidade óssea após os 50 anos de idade.

- Pacientes em uso prolongado de glicocorticóides por via oral ou parenteral.

- História e/ou achados sugestivos de fratura vertebral não documentada por estudo radiológico prévio.

- O diagnóstico de fratura deve basear-se em avaliação visual e incluir indicação de grau/gravidade, utilizando-se os critérios de avaliação semiquantitativa (SQ) desenvolvidos por Genant e cols. (14).

- A decisão de recomendar ou realizar estudos complementares adicionais deve fundamentar-se no quadro clínico de cada paciente, incluindo os resultados de VFA.

\section{ULTRASSONOGRAFIA QUANTITATIVA (QUS)}

- O único sítio validado para o uso clínico da QUS em osteoporose é o calcâneo.

- Equipamentos de QUS de calcâneo validados são capazes de estimar fratura por fragilidade em mulheres na pós-menopausa e em homens com idade superior a 65 anos, independentemente da DMO medida em equipamentos de DXA central.

- A correlação entre os resultados obtidos por QUS e DXA central é moderada.

- QUS do calcâneo associada a fatores de risco clínicos podem ser usados para identificar população com baixa probabilidade de fratura na qual avaliação adicional não seja mais necessária.

- Medidas de DXA central na coluna e no fêmur constituem o método de escolha para a tomada de decisão terapêutica. Entretanto, se a DXA central não for acessível, o tratamento farmacológico pode ser iniciado se a probabilidade de fratura, avaliada pelo QUS do calcâneo com pontos de corte específicos, associados a fatores de risco clínicos, for suficientemente alta.

- Equipamentos QUS não são clinicamente úteis no monitoramento de efeitos esqueléticos dos medicamentos disponíveis para o tratamento da osteoporose.

\section{DIAGNÓSTICO EM CRIANÇAS E EM ADOLESCENTES (IDADE INFERIOR A 20 ANOS)}

- T-escores não devem ser utilizados, não devem sequer aparecer nos relatórios ou nos impressos DXA; utilizar sempre Z-escore.

- O diagnóstico de osteoporose não pode ser feito fundamentando-se unicamente no critério densitométrico; o diagnóstico requer o antecedente de uma fratura de osso longo de membro inferior, compressão vertebral ou duas ou mais fraturas de extremidades superiores, associado à DMO baixa.

- Terminologias como "baixa massa óssea para a idade cronológica" ou "abaixo da faixa esperada para a idade" podem ser utilizadas se o Z-escore for menor que -2 DP. Não utilizar o termo osteopenia.

- Z-escores devem ser interpretados à luz do melhor banco de dados pediátrico possível, ajustado para a idade. A base de dados utilizada deve ser citada no relatório.

- A coluna lombar em PA e o corpo total (preferencialmente sem incluir a DMO medida na cabeça - CIMC) são os sítios mais exatos e reprodutíveis para se avaliar o CMO e a DMO areal.

- Embora faltem dados para se estimar com precisão o risco de fraturas em crianças e adolescentes por meio da DMO, estudos recentes demonstram que a DMO do corpo total baixa e a área óssea em relação à altura são parâmetros associados ao maior risco nesta população (15).

- Não existe consenso em relação aos ajustes da DMO para fatores, como tamanho do osso, estadiamento puberal, maturidade esquelética e composição corporal.

- Medidas seriadas de DMO devem ser realizadas na mesma máquina, usando o mesmo modo de aquisição e versão de software. No entanto, alterações desses parâmetros podem ser necessárias com o crescimento do indivíduo.

\section{AVALIAÇÃO DXA EM CRIANÇAS E ADOLESCENTES COM DOENÇAS CRÔNICAS COM COMPROMETIMENTO ESQUELÉTICO}

- DXA é parte de uma ampla avaliação da saúde óssea em pacientes pediátricos com risco de fratura aumentado.

- Intervenções terapêuticas não devem ser instituídas fundamentando-se exclusivamente em uma medida isolada da massa óssea à DXA.

- Realizar DXA antes e durante tratamento com drogas com repercussão na massa óssea.

- Pacientes com doença óssea primária (por exemplo, osteogênese imperfecta) ou potencial doença óssea secundária (por exemplo, doenças inflamatórias crônicas, dis- 
túrbios endocrinológicos, história de câncer na infância ou transplante prévio [não-renal]), CMO e DMO areal de coluna lombar e CIMC devem ser medidos no momento de apresentação clínica da doença.

- Nos pacientes com talassemia maior, a medida do CMO e da DMO areal de coluna e CIMC estão indicados na presença de fratura ou aos 10 anos de idade.

- Em crianças sob imobilização crônica (por exemplo, paralisia cerebral), CMO e DMO areal de coluna lombar e CIMC devem ser medidos no momento de apresentação da fratura.

- DXA não deve ser realizado na presença de dor, espasmo ou contratura, que impeçam o posicionamento do paciente na mesa de exames ou que propiciem demasiado desconforto ao paciente.

- O intervalo mínimo para se repetir a densitometria óssea no monitoramento de tratamento com droga osteoativa ou doença nesta população é de seis meses.

\section{INTERPRETAÇÃO E RELATÓRIO DE DXA EM CRIANÇAS E EM ADOLESCENTES}

- Medições da massa magra e massa de gordura na aquisição de corpo inteiro podem ser úteis na avaliação de pacientes com condições crônicas associadas à desnutrição (por exemplo, anorexia nervosa, doença inflamatória intestinal, fibrose cística) ou déficit muscular e esquelético (como na osteoporose idiopática juvenil).

- O quadril (fêmur proximal) não é sítio confiável em pacientes em crescimento por causa da significativa variabilidade no desenvolvimento do esqueleto e da ausência de ROI reprodutíveis nesse sítio.

- Em crianças com atraso do crescimento linear ou de maturação sexual, os resultados de CMO e DMO areal da coluna e do CIMC podem ser ajustados para altura, ou idade/altura, ou comparados aos bancos de dados de referência pediátricos que forneçam Z-escores específicos para idade, sexo e altura.

- Os bancos de dados de referência devem incluir amostra da população sadia suficientemente grande para caracterizar a variabilidade normal das medidas ósseas nesta faixa etária, levando em consideração sexo, idade e etnia.

- A reavaliação deve ser realizada somente quando a variação esperada na DMO areal seja igual ou exceda a MVS.

- É recomendável que o médico solicitante forneça informações sobre o estágio puberal do paciente, parâmetro muito importante para a interpretação de resultados.

\section{PQCT EM CRIANÇAS E EM ADOLESCENTES}

- Os dados de referência são insuficientes para o uso clínico de $\mathrm{pQCT}$ para estimativas de risco de fratura.

- Na avaliação do antebraço, o lado não-dominante deve ser examinado.

- Os sítios de medida devem incluir a metáfise e a diáfise óssea.

- A determinação do erro de precisão, cálculo da MVS e definição do intervalo para monitoramento devem ser realizados, como já descrito para a DXA.

\section{NOMENCLATURAS EM DENSITOMETRIA}

- DXA - não DEXA.

- VFA - não LVA/IVA ou IVA-HD.

- T-score - não T Score; T-escore ou t score.

- Z-score - não Z Score; Z-escore ou z score.

\section{CASAS DECIMAIS PARA VALORES DXA}

- DMO: 3 casas decimais (por exemplo, 0,927 g/ $\mathrm{cm}^{2}$ ).

- T-escore: 1 casa decimal (por exemplo, -2.3).

- Z-escore: 1 casa decimal (por exemplo, 1.7).

- CMO: 2 casas decimais (por exemplo, $31,76 \mathrm{~g}$ ).

- Área: 2 casas decimais (por exemplo, 43,25 cm²).

- Percentuais em relação aos dados de referência: número inteiro (por exemplo, 82\%).

\section{GLOSSÁRIO}

CIMC - corpo inteiro menos a cabeça.

CMO - conteúdo mineral ósseo.

DMO - densidade mineral óssea.

DXA - absorciometria por dupla emissão de raios X.

MVS - mínima variação significativa.

OMS - Organização Mundial de Saúde.

PA - póstero-anterior.

pDXA - absorciometria periférica por dupla emissão de raios $\mathrm{X}$.

pQCT - tomografia quantitativa computadorizada periférica.

QCT - tomografia quantitativa computadorizada.

QUS - ultrassonometria quantitativa.

ROI - região de interesse.

vDMO - DMO volumétrica.

VFA - vertebral fracture assessment.

Declaração: Os autores declaram não haver potencial conflito de interesse científico. 


\section{REFERÊNCIAS}

1. Gracia CR, Sammel MD, Freeman EW, Lin H, Langan E, Kapoor S, Nelson DB. Defining menopause status: creation of a new definition to identify the early changes of the menopause transition. Menopause. 2005;12(2):117-120.

2. Finkelstein JS, Brockwell SE, Mehta V, Greendale GA, Sowers MR, Ettinger $\mathrm{B}$, et al. Bone mineral density changes during the menopause transition in a multiethnic cohort of women. J Clin Endocrinol Metab. 2008;93:3861-8.

3. Kanis JA, Oden A, Johnell O, Johansson H, De Laet C, Brown J, et al. The use of clinical risk factors enhances the performance of BMD in the prediction of hip and osteoporotic fractures in men and women. Osteoporos Int. 2007;18(8):1033-46.

4. Kanis JA, Johansson H, Oden A, Johnell O, De Laet C, Melton LJ, et al. A meta-analysis of prior corticosteroid use and fracture risk. J Bone Miner Res. 2004;19:893-9.

5. Kanis JA, Johnell O, De Laet C, Johansson H, Oden A, Delmas $P$, et al. A meta-analysis of previous fracture and subsequent fracture risk. Bone. 2004;35:375-82.

6. Johnell O, Kanis JA, Oden A, Johansson H, De Laet C, Delmas P, et al. Predictive value of bone mineral density for hip and other fractures. J Bone Miner Res. 2005;20:1185-94.

7. De Laet C, Kanis JA, Oden A. Body mass index as a prediction of fracture risk. A meta-analysis. Osteoporos Int. 2005;16(11):1330-8.
8. Kanis JA, Johansson H, Johnell O, Oden A, De Laet C, Eisman A, et al. Alcohol intake as a risk factor for fracture. Osteoporos Int. 2005;16:737-42.

9. Kanis JA, Johansson H, Oden A, De Laet C, Johnell O, Eisman JA, et al. A meta-analysis of milk intake and fracture risk: low utility for case finding. Osteoporos Int. 2005;16(2):155-62.

10. Kanis JA, Johnell O, Oden A, Johansson H, De Laet C, Eisman JA, et al. Smoking and fracture risk: a meta-analysis. Osteoporos Int. 2005;16:222-8.

11. Pinheiro MM, Ciconelli RM, Martini A, Ferraz MB. Clinical risk factors for osteoporotic fractures in Brazilian women and men: the Brazilian Osteoporosis Study (BRAZOS). Osteoporos Int. [acessado em jul 2008]; Disponível em: http://www.cdc.gov/nchs/products/elec_prods/ subject/nhanes3.htm.

12. WHO Study Group. Assessment of fracture risk and its implication to screening for postmenopausal osteoporosis. Report of a WHO Study Group. 1994 World Health Organ Tech Rep Ser. 843:1-129.

13. Shepherd JA, Morgan SL, Lu Y. Comparing BMD results between two similar DXA systems using the generalized least significant change. J Clin Densitom. 2008;11(2):237-42.

14. Genant HK, Li J, Wu CY, Shepherd JA. Vertebral fractures in osteoporosis: a new method for clinical assessment. J Clin Densitom. 2000;3(3):281-90.

15. Clark EM, Ness Ar, Bishop NJ, Tobias JH. Association between bone mass and fractures in children: a prospective cohort study. J Bone Miner Res. 2006;21:1489-95. 